\title{
CRITERIA AND INDICATORS OF FORMATION OF HUMANISTIC AND VALUE ATTITUDE OF PRIMARY SCHOOLCHILDREN TOWARDS THEMSELVES
}

\author{
Oksana Holiuk \\ Vinnytsia Mykhailo Kotsiubynskyi State Pedagogical University, Ukraine \\ Natalia Pakhalchuk \\ Vinnytsia Mykhailo Kotsiubynskyi State Pedagogical University, Ukraine \\ Nataliia Rodiuk \\ Vinnytsia Mykhailo Kotsiubynskyi State Pedagogical University, Ukraine \\ Olena Demchenko \\ Vinnytsia Mykhailo Kotsiubynskyi State Pedagogical University, Ukraine \\ Galina Kit \\ Vinnytsia Mykhailo Kotsiubynskyi State Pedagogical University, Ukraine
}

\begin{abstract}
Scientific works show that it is necessary to solve the humans problem of the formation of values and value attitude. Early school age is a period of active development of ideological orientations, interests, behavioral strategies, assessments and self-evaluation. The purpose of the study lay in developing and testing the diagnostic tools of the formation of a humanistic and value attitude of the schoolchildren towards themselves and in determining on this basis the appropriate pedagogical conditions for improving the investigated quality. The primary schoolchildren ( $n=317$, age 8-9) took part in the study. The results of the preliminary stage of the pedagogical experiment revealed the need to raise the level of humanistic and value attitude of children (low level - 61,5\%, average level - 30,9\%, high level - 7,6\%). Primary school teachers are important organizers of the educational process with students, but they do not use enough methods that are effective in solving this research problem. In order to humanize the environment in primary school, authors suggest using game exercises, conversations, elements of training, including children in various types of socially significant activities, creating axiological educational situations, etc.
\end{abstract}

Keywords: humanistic and value attitude, levels of formation of humanistic and value attitude, pedagogical conditions, primary schoolchildren.

\section{Introduction}

An important issue today is the study of patterns of formation, establishment and development of a conscious, active, creative person. New trends in the development of Ukrainian society, qualitative transformation of various life 
aspects, of changes in the content and nature of the material and spiritual conditions of life, forming radically different economic and sociopolitical environment - all this cannot affect the outlook of the individual. There are serious changes in the structure of youth value orientations in the new social conditions. Changes in the value orientations of youth do not occur suddenly, this is preceded by a number of preconditions. Changes in the society, in the material and spiritual sphere, require new approaches to the analysis of spiritual values.

The main element in the worldview formation are value orientations. They determine the interaction, struggle and harmonization of interests, requirements, criteria, regulate the behavior, defining the strategy of social life of the individual. At the same time, it is important not only to develop a system of values that allow a person to adapt to a new society quickly and actively - it is important not to lose own national ideals, moral values, cultural traditions of the society.

The purpose of the article is to analyze the current state of formation of humanistic and value attitude of the primary schoolchildren towards themselves. According to the purpose, the task of the study is defined: to distinguish the structural components of humanistic and value attitude towards oneself; to define criteria, indicators and levels of formation of humanistic and value attitude of the primary schoolchildren towards themselves; to determine the pedagogical conditions of formation of humanistic and value attitude of the primary schoolchildren towards themselves.

Research methods: to obtain empirical data, in order to identify the levels of humanistic and value attitude of children, was used targeted follow-up of children in various activities, pedagogical analysis, interviewing, clarifying conversations, design methodology «Diagnosis of emotional and value attitude towards oneself» by A. Pryhozhan and Z. Vasyliauskaite (Shchetinina, 2000), the method of "Acceptance of oneself», expert evaluation, questionnaire «Self-determination in life situations», which contributed to the objective study of the state of the problem in the practice of modern primary school.

\section{The theoretical background}

An important aspect of the study of the value orientations of youth in the new conditions is the analysis of the system of human values. The concept of «value» and «value orientation» arose on the verge of several areas - philosophy, axiology, social and cultural anthropology, sociology, and psychology.

The category of value is one of the most difficult categories. In the definition of this category in relation to its nature, role, meaning for various aspects of life and human behavior there are contradictory judgments. This is due to the fact that this category is not only theoretical, but reflects the real criteria, benchmarks for behavior and actions of the individual. 
According to scientists (Hitlin \& Piliavin, 2004), values express different motivational goals and make it possible to understand the links between previous social positions and individual choices that serve to recreate aspects of a social structure.

The definition "value» reveals the attitude of man to the world, which concerns not so much intellectual as the affective side. The value is as if distributed between a person and the surrounding environment and exists in their interaction. The bearer of value can be any personality, object, phenomenon, which is in interaction with the consciousness of a personality.

Values displayed in human consciousness form value orientations. Values are a life goal and ideological orientations that are formed in the process of comparing personal experience, culture and traditions. Value orientations of the person are studied in the context of pedagogical diagnostics, are considered in tight connection with the spiritual culture, spiritual needs, interests, evaluative judgments. Different variants of the research of values and value attitude exist: ranging, rating, hierarchical linear models, structural equation test for value and behavioral attitude and behavioral hierarchy (Homer \& Kahle, 1988; Boninger, Krosnick, \& Berent, 1995; Hitlin \& Piliavin, 2004).

Formation of needs and values comes from childhood under the influence of various factors and causes. The development of a person depends on the environment in which the child is located, who surrounds her, which priorities are preferred. Researchers underline that parents, peer groups, professional circles and culture can serve as sources of values in general. Meanwhile some values can only be taken as social norms. However, a positive self-attitude, activation of the concept «oneself» will lead to the assimilation of values (Verplanken \& Holland, 2002).

Neff K. considers an alternative conceptualization of a healthy attitude towards oneself in the form of self-affirmation, which corresponds to the humanistic value of man (Neff, 2003). It is like self-pardon, but it is not identified with passivity and inactivity with respect to the shortcomings that are observed in oneself. Positive and emotional attitude includes feelings of kindness and caring for oneself and others (Nelson, Hall, Anderson, Birtles, \& Hemming, 2017). Moore J. and Smith M. demonstrate the degree of self-esteem dependency on performing daily evaluations of oneself and others, and the impact of others' assessments on social and emotional well-being (Moore \& Smith, 2018).

Scientists investigate various aspects of value attitude: to computer training (Vekiri \& Chronaki, 2008), to the environment (Onur, Sahin, \& Tekkaya, 2012), etc. The research of humanistic and value attitude to oneself becomes actual one.

An important factor in the spiritual development of the child is the formation of value orientations on a humanistic basis. Therefore, its worldview, which is the 
basis for shaping the system of humanistic and value-based attitude of the child to himself, becomes important in the formation of the values of the individual.

The definition «humanistic-value attitude of the child to himself» in our research is interpreted as a relatively resistant system of values expressed in the child's ability to empathy, awareness and perception of the surrounding reality through the prism of their own evaluative judgments based on cognitive, emotional and evaluative, behavioral components.

\section{Methodology, organization and results of the research}

The results of the preliminary stage of the pedagogical experiment presented in this paper are found to be a part of a broader study conducted in primary schools in Vinnytsia region (Ukraine). The ultimate goal of the study is the scientific substantiation and experimental verification of the effectiveness of the pedagogical conditions for the formation of a humanistic and value attitude of the primary schoolchildren towards themselves. At the stage of the statement, 317 primary schoolchildren of 3-4 classes in both urban and rural schools took part in the experiment.

Diagnostic examination of the formation of the primary schoolchildren of humanistic and value attitude towards themselves was made in each individual component (cognitive, emotional and evaluative, behavioral ones).

For the quantitative processing of the results of diagnostics, we have developed a mechanism for assessing the level of formation of humanistic and value attitude of the primary schoolchildren towards themselves. According to the results of each diagnostic procedure, the degree of manifestation of a certain indicator for each criterion correlated with the content (qualitative) characteristics of the levels of formation humanistic and value relationship of the child to himself and was evaluated at a five-point scale. At the end of each index, its numerical value $P_{m . n}$ was found as the arithmetic mean of the points obtained, where $m$ is the serial number of the criterion, $\mathrm{n}$ is the ordinal number of the indicator for this criterion. If $0 \leq \mathrm{P}_{\mathrm{m} . \mathrm{n}}<2$, we believed that the respondent had a low level of manifestation of this indicator, if $2 \leq \mathrm{P}_{\mathrm{m} . \mathrm{n}}<4$ - average level, if $4 \leq \mathrm{P}_{\mathrm{m} . \mathrm{n}} \leq 5$ - high one.

In order to determine the level of formation of each structural component of the value attitude of the primary school student towards himself, we transferred the data received for each respondent for each indicator into standard scores: the high level of the indicator was estimated at 2 points, the average -1 point, the low -0 points. The coefficient of formation of each component of the value attitude $K_{\mathrm{m}}$ was calculated by the formula 1 created by the authors: 
SOCIETY. INTEGRATION. EDUCATION

Proceedings of the International Scientific Conference. Volume III, May $22^{\text {th }}-23^{\text {th }}, 2020.198-211$

$$
K_{m}=\sum_{k=1}^{n} K_{m \cdot n},
$$

where $\mathrm{n}$ - number of indicators according to which the diagnostic study was conducted,

$\mathrm{K}_{\mathrm{m} . \mathrm{n}}-$ number of points obtained for each of the $\mathbf{n}$ indicators (high -2 points, the average -1 point, low -0 points).

Depending on the received quantitative value of the coefficient $K_{\mathrm{m}}$ the level of formation of the corresponding component was determined:

High level $-5 \leq K_{\mathrm{m}} \leq 6$;

The average level $-2 \leq \mathrm{K}_{\mathrm{m}}<5$;

The low level $-0 \leq K_{\mathrm{m}}<2$.

Using the analogous procedure, we determined the numerical coefficient of the level of formation of the value attitude $\left(K_{\mathrm{v}}\right)$ of the primary school student towards himself as to a holistic entity. For this, the coefficients of the formation of the components of $K_{\mathrm{m}}$ were repeatedly converted to standard points depending on the determined level (high -2 points, average -1 points, low -0 ), and numerical value of the coefficient $K_{v}$ was found for each respondent according to the formula 1.

The study and analysis of the theoretical basis on the subject of research has given us the grounds for the concept of humanistic and value attitude of the primary pupil towards himself as a relatively stable system of values, expressed in the ability of the child to the holistic experience, awareness and perception of the surrounding reality through the prism of their own evaluative judgments based on cognitive, emotional and evaluative, behavioral components. For each component we have identified diagnostic criteria and relevant indicators.

Thus, we consider the criterion of the cognitive component to be humanistic and valuable awareness of the child, whose indicators are: awareness of the personality of his value as a Human; a system of ideas about worthy and unworthy behavior; value and meaning formations associated with the image of «I am». The criterion of the emotional and evaluative component is the emotional and value settings in the relationship interactions, the indicators of which are: experience of the personality of his value; emotional attitude to situations that affirm dignity, respect for comrades; adequacy of self-esteem. The criterion of a behavioral component is characterized by the presence of a stable humane life position; its indicators are the person's desire for development; the ability to regulate behavior according to a value relation to oneself and others.

Based on the distinguished criteria and indicators, we have identified the levels of formation of humanistic and value attitude of primary pupils toward 
themselves (high, medium, low). Here is a description of the identified levels in the context of the formation of each component (cognitive, emotional and evaluative, behavioral ones):

High level:

These students are characterized by the fact that their humanistic and value orientations are aimed at themselves and other people; they respect, love and appreciate themselves, acquaintances and strangers and their loved ones. They formulated a system of decent and unworthy behavior; value and meaning entities that are associated with the image of «I am» are manifested. They worry not only about their values, a situation that affirms dignity, but also respect their comrades, listen to themselves and respect the opinions of adults; self-esteem is characterized by adequacy. They strive for self-development, are able to set themselves humanistic goals, to regulate behavior in accordance with the value relation to themselves and to others.

Average level:

The humanistic and value orientations of these children are aimed at moral and valuable life guidance, search and choice of life path; they do not despair, they value everything that gives them life. However, they are not sufficiently aware of their own value, but differentiate their negative and positive features; the system of decent and unworthy behavior in them is not sufficiently formulated; value and meaning entities that are related to the image «I'm» not clearly expressed. Insufficiently expressing their values, a situation that confirms dignity, a respectful attitude to their comrades is situational; more often or only listen to themselves, and adult thoughts are taken into account in most cases from the point of view of their own benefit, or they fully submit to the position of an adult without trying to express their own position. Self-esteem, as a rule, is slightly overestimated (sometimes - understated). They do not always strive for selfdevelopment, they are able to set goals, but not always these goals have a humanistic and value orientation; aspire to regulate behavior in accordance with the value relation to themselves and to others, but sometimes they do not succeed.

Low level:

Valuable orientations of these children are aimed only at material values. They do not realize their value at all; the system of decent and unworthy behavior in them is not formulated; value and meaning entities that are related to the image «I am» have not expressed at all. Not expressing their values, a situation that affirms dignity, almost do not show a respectful attitude to their comrades. They neither listen nor to themselves, nor to the opinion of adults. Often show aggressiveness. Their self-esteem is inadequate. Do not seek self-development, their life goals are not humanistic and value orientation. They do not know how to regulate behavior in accordance with the value relation to themselves and to others. 
To study the condition of the formation of the cognitive component of the humanistic and value relation to ourselves, we have developed a questionnaire «I am a Man!» which contains 12 questions and unfinished sentences aimed at identifying the humanistic and value awareness of children. The first five questions are aimed at determining the level of awareness of the personality of his value as a Person; four following - a system of ideas about worthy and unworthy behavior; the last three are the nature of value and meaning entities associated with the image of «I am».

1. I think that as a Human, I am capable of ...

2. I think that I as a person worthy ...

3. «Honor others» means ...

4. «Respect yourself» means ....

5. For me, the honor is ...

6. What, in your opinion, is worthy of a person: what are his actions, manifestations of which qualities?

7. And what is unworthy? Who is called a scumbag? For what?

8. Is it possible for you to always be a worthy man who is respected by everybody?

9. What prevents you from becoming such a person? What should you do in your character?

10. The true Person is ...

11. The main aim of Person...

12. In my life, I adhere to the following rules: ...

Upon completion of the survey, we conducted refining conversations, during which the children were invited to continue the sentence:

1. I am a person because I always ...

2. I am a person and therefore never ...

3. When I'm offended, I ...

4. I know that I'm unlike others because ...

The analysis of the results divided all respondents into three groups. To the first group (high level) 34 respondents (10,7\%) were assigned. These students are characterized by the fact that their humanistic and value orientations are aimed at themselves and other people; they respect, love and appreciate themselves, acquaintances and strangers and their beloved. They formulated a system of decent and unworthy behavior; value and meaning entities are associated with the image of «I am».

We included 117 respondents to the second group (36,9\%). Their humanistic and value orientations and moral and value-oriented guidelines of life aimed at finding and choosing a way of life; they do not despair, they value everything that gives them life. However, they are not sufficiently aware of their own value, but differentiate their negative and positive features; the system of decent and 
unworthy behavior in them is not sufficiently formulated; value and meaning entities that are related to the image I'm not clearly expressed.

The third group includes children whose value orientations are aimed only at material values. Such were 166 respondents (52,4\%). They do not realize their value at all; the system of decent and unworthy behavior in them is not formulated; value and meaning entities that are related to the image «I am» were not expressed at all.

The obtained results show the predominance of low level of humanistic and valuable awareness of primary pupils; many children do not have the need for self-knowledge and self-analysis, without which there can be no full sense of life, worthy of a Human. There is a mass of consciousness under the influence of very similar and depleted needs of children. The lowest results relate to the love of people and respect for others.

In order to determine the state of formation of the emotional and evaluative component of early school-aged children, we studied the emotional and value settings in relation interactions. Diagnostics of the depth of experience of the personality of his value was carried out with the help of the design method A. Pryhozhan and Z. Vasyliauskaite (Shchetinina, 2000). During the implementation of this technique, the children drew three pictures: «Bad boy/girl», «Beautiful boy/girl», «I am».

Analysis of the drawings showed that most children had a positive attitude towards themselves (drawing «I am»), using warm colors. The children tried to portray themselves in the center, which testifies to an adequate self-esteem, trying to explain why on the one picture the boy (girl) is bad, and on the other is good. The final conclusion was made only after clarifying conversations.

The study of emotional attitude to the phenomenon of human dignity and subjectively related concepts (the situation of self-affirmation and humiliation of dignity) and respectful attitude to friends took place in the process of purposeful pedagogical supervision of children in various activities (educational, extracurricular, game, under time of informal communication with peers, with relatives, with teachers, etc.), as well as in interviews with teachers (class teacher, teacher of music, teacher of English language, psychologist). It should be noted that the results of these diagnostic procedures have shown that children can experience their values, but at the same time self-asserting and degrading their dignity.

Establishing the degree of adequacy of self-assessment for early school-aged children was through the implementation of the method "Acceptance of oneself», which is aimed at determining the positive and negative qualities of the individual, their individual characteristics; the ability to recognize their disadvantages and demonstrate pluses, tolerates and understands others who also have their drawbacks and dignity. 
The children were offered to divide a sheet of paper into two halves. On the left, in the column «My shortcomings», it was necessary to record everything honestly that a child considers their shortcomings today, now, at the moment.

After that, the children were given the following instruction: «In front of each drawback that you mentioned and listed, in the column «My Dignity» write one of its merits, something that can be countered by the disadvantages that you have at the moment». For each stage of work was given 5 minutes. Upon completion of the methodology with children, specific conversations were held. In addition, the children had to finish the sentence: «My relationships with classmates can be described as ...», «Most of all I value in my classmates ...». As a result of all the above mentioned procedures, it turned out that only.

37 respondents $(11,7 \%)$ are not only experiencing their values, a situation that confirms dignity, but also respect their comrades. They listen to themselves and respect the opinions of adults; self-esteem is characterized by adequacy. Therefore, we interpret their emotional and value settings in relation interactions as having a high level.

156 respondents $(49,2 \%)$ do not have enough of their values, a situation that confirms dignity, a respectful attitude to their comrades is situational. They are more likely to listen only to themselves, and adult thoughts are taken into account in most cases from the point of view of their own benefit, or they fully submit to the position of an adult without trying to express their own position. Self-esteem, as a rule, is somewhat overestimated (sometimes - understated). Therefore, we interpret their emotional and value settings in relation interactions as having an average level.

124 respondents $(39,1 \%)$ do not worry about their values, the dignity situation almost does not show a respectful attitude to their comrades. They do not listen neither themselves, nor the thoughts of adults. Often show aggressiveness. Their self-esteem is inadequate. Therefore, we interpret such emotional and value settings in relation interactions as having a low level.

Thus, according to the study, for the majority of early school-aged children, the low level of emotional and value facilities in the intercourse interactions is characteristic, which testifies to the low level of formation of the emotional and evaluated component of the humanistic value relation to oneself.

To find out the state of formation of the behavioral component of the humanistic and value attitude towards themselves, we offered a questionnaire «Self-determination in life situations», questions are aimed at determining the presence of a stable, humane attitude of the primary schoolchildren.

Since the aspiration of the individual for development involves the ability to set goals for ourselves, we have proposed to children the questionnaire «Polifactor assessment of vital values». Questionnaire questions allow to evaluate the vital values of the primary schoolchildren based on the following factors: the factor of 
self-realization, external success, service to society, protection of one's own Self, full life, strength and independence, friendship, wealth and health.

The analysis of the results showed that early school-aged children aspire to self-realization, full-fledged life, ready to serve society, want to have strength and independence, set goals and are ready for self-development.

The results of the children's responses to the questionnaire's questions were corrected in the process of targeted monitoring of children in various activities, which allowed investigating the practical skills of primary pupils to regulate behavior in accordance with the value relation to themselves and others.

As a result of the implementation of all the above diagnostic procedures, the following conclusions were made regarding the presence of a stable, humane attitude of the primary schoolchildren. The high level of its formation was recognized by 24 respondents $(7,6 \%)$, as they seek self-development, are able to set themselves humanistic goals, to regulate behavior in accordance with the value relation both to oneself and to others. The average level - 98 respondents (30,9\%). These children do not always strive for self-development; know how to set goals, but not always these goals have a humanistic value orientation. They want to regulate behavior in accordance with the value relation both to themselves and to others, but sometimes they do not succeed. The low level is characteristic of 195 respondents (61,5\%). They do not seek self-development, their life goals are not humanistic and value orientation. They do not know how to regulate behavior in accordance with the value relation to themselves and to others.

The obtained results indicate that many children do not have the desire for development, there is a mass in the regulation of behavior in accordance with the value relation not to oneself, but to others.

The study of humanistic and value awareness, emotional and value settings in relation interactions, the presence of a stable, humane attitude among the primary schoolchildren made it possible to draw conclusions about the levels of formation of the components of their humanistic and value attitude towards oneself, namely, cognitive, emotional and evaluative, behavioral ones (Table 1).

Table 1 Levels of formation of components of humanistic and value attitude of the primary schoolchildren towards themselves

\begin{tabular}{|l|c|c|c|c|c|c|}
\hline & \multicolumn{2}{|c|}{ High level } & \multicolumn{2}{c|}{ Average level } & \multicolumn{2}{c|}{ Low level } \\
\hline & amount & $\%$ & amount & $\%$ & amount & $\%$ \\
\hline Cognitive component & 34 & 10,7 & 117 & 36,9 & 166 & 52,4 \\
\hline $\begin{array}{l}\text { Emotional and evaluative } \\
\text { component }\end{array}$ & 37 & 11,7 & 156 & 49,2 & 124 & 39,1 \\
\hline Behavioral component & 24 & 7,6 & 98 & 30,9 & 195 & 61,5 \\
\hline
\end{tabular}


The generalization of quantitative and qualitative research results for all three components showed that a high level of humanistic and value attitude formation is available to 31 respondents $(9,8 \%)$; the average is 139 respondents (43,8\%); low - in 147 respondents (46,4\%) (Fig. 1).

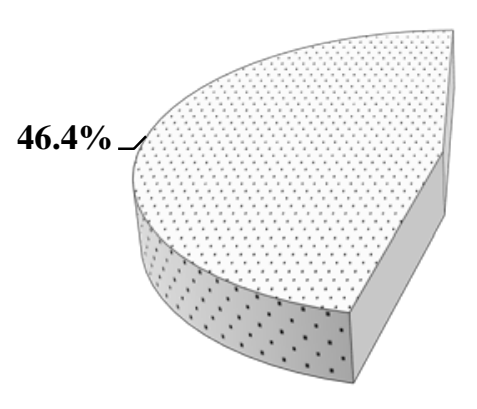

SHigh level

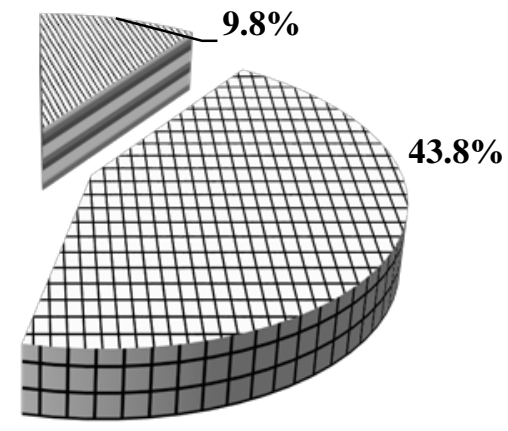

¿Low level

Figure 1 Generalized result of the diagnostics of the level of formation of the humanistic and value attitude of the primary schoolchildren towards themselves

The synthesis of the materials of the staging phase of the experiment showed that teachers are aware of the importance of forming a value attitude of the primary schoolchildren towards themselves. However, they do not use enough methods that are effective in solving this problem. The educational work is limited in familiarizing children with game exercises and activities aimed at fostering a friendly relationship between children. The cooperation of educational institutions and parents on this problem is unsatisfactory.

\section{Discussion}

On the basis of our research, we determined the pedagogical conditions for the formation of the humanistic and value attitude of the child towards himself:

1. Humanization of the environment in primary school, which involves increasing attention to the student's personality as the highest value of society.

2. Organization of the subject-subjective interaction of participants in the educational process, which requires from the teacher to treat a child as a smart partner; enrich the world of a child with positive emotions; recognize the fullness, subjectivity and equality of the participants in the interaction.

3. Use of a positive example of moral behavior presented in oral folk art, fiction and real life, in order to influence emotions, feelings of children, 
and to develop positive and value attitude towards themselves, actions and deeds of other people.

4. Creation of axiological educational situations, during which the child faces with a moral choice, carrying out the process of internalization and introspection of humanistic values.

5. Inclusion of children in various forms of socially significant activities aimed at helping peers, relatives, acquaintances, people with special educational needs or the elderly, active participation in which will promote the transformation of humanistic feelings in the development of a stable humane life attitude.

6. Using the game exercises, conversations, elements of training to activate reflexive processes, development of the ability to self-assess of values and moral self-education of self-development.

An important condition for the effectiveness of the implementation of the spiritual development of the child, formation of child's value orientations is the preparedness of teachers for this educational work on humanistic principles and develops their ability to professional self-improvement, developing a subject position (Demchenko, Kit, Holiuk, \& Rodiuk, 2018). In the process of organization of educational activity at the universities future teachers should be aware of the basic principles of the subject-subjective approach in education, to learn the system of psychological and pedagogical knowledge about the value as a scientific phenomenon, the peculiarities of the formation of semantic-oriented sector in the early school age. The mastery of modern personality-oriented technologies and methods of forming axiological development of pupils of primary school is significant, in particular they are methods of conducting trainings, creation of educational situations, techniques of collective gaming communication (Lapshyna \& Lyubchak, 2019).

\section{Conclusions}

1. Theoretical and methodological analysis of literary sources made it possible to determine the components (cognitive, emotional and evaluative, behavioral) of humanistic and value attitude of primary schoolchildren towards themselves.

2. To conduct the experimental research, we were offered criteria (humanistic and value awareness, emotional and value settings in relation interactions, the presence of a stable humane position of life) and indicators of the investigated quality.

3. Analysis of the results of the ascertaining stage of the study showed the predominance of low level of its formation $(46,4 \%)$ by all the components we identified. The substantiation of pedagogical conditions was made on the 
basis of this research. In our opinion, in order to form humanistic and value attitude towards themselves by primary schoolchildren, it is necessary to create a partnership environment, provide subjectivity in communication, demonstrate the samples of moral behavior, organize socially significant activities and educational situations of choice, activate reflexive mechanisms of individual.

\section{Summary}

At the stage of the technocratization and informatization of society, the axiological component is the basis of the humanization of society and the education of man as a person, a citizen, a patriot. Values and value orientations are important phenomena that have an interdisciplinary status and are studied within the framework of philosophy, axiology, social and cultural anthropology, sociology, psychology. The formation of needs and values is a complex dialectical process that begins in the early years under the influence of various factors. The early school age is sensitive to the assimilation of humanistic settings and value orientations, the development of habits and skills of moral behavior in general and, above all, the formation of a humanistic and value attitude of the child towards himself. Theoretical and methodological analysis gave an opportunity to substantiate the content of the value and orientative approach in the educational process of the primary school, which involves the synthesis of cognitive, emotional and evaluative, behavioral components. Planned and conducted ascertaining experiment revealed the dominance of the low level of formation of the humanistic and value attitude towards themselves by the primary school students. In view of this, there is a need to create special conditions in the educational process of the school for the forming the axiological sphere of children.

\section{References}

Boninger, D.S., Krosnick, J.A., \& Berent, M.K. (1995). Origins of attitude importance: Selfinterest, social identification, and value relevance. Journal of Personality and Social Psychology, 68, 61-80.

Demchenko, O., Kit, G., Holiuk, O., \& Rodiuk N. (2018). Development of future teachers' subjectivity in the context of preparation for working with gifted children. Society. Integration. Education, II, 507-519. DOI: http://dx.doi.org/10.17770/sie2018vol1.3167

Goluk, O., Gaydukevich, A., Pakhal'chuk, N., Karuk, I., \& Kolesnik, K. (2018). Education of children's self-esteem as a projection of moral and humanistic values. Science Review, 1(8), 5, 13-17.

Hitlin, S., \& Piliavin, J.A. (2004). Values: Reviving a dormant concept. Annual Review of Sociology, 30, 359-393.

Homer, P.M., \& Kahle, L.R. (1988). A structural equation test of the value-attitude-behavior hierarchy. Journal of Personality and Social Psychology, 54, 638-646.

Lapshyna, I., \& Lyubchak, L. (2019). Using the Technology of Collective Game Communication in the Process of Forming the Diamonological Competency of Primary School Pupils. Society. Integration. Education, II, 267-279. DOI: http://dx.doi.org/10.17770/sie2019vol2.3995

Moore, J.S.B., \& Smith, M. (2018). Children's levels of contingent self-esteem and social and emotional outcomes. Educational Psychology in Practice, 34(2), 113-130. 
Holiuk et al., 2020. Criteria and Indicators of Formation of Humanistic and Value Attitude of Primary Schoolchildren Towards Themselves

Neff, K. (2003). Self and identity. Taylor \& Francis, 2, 85-101.

Nelson, J.R., Hall, B.S., Anderson, J.L., Birtles, C., \& Hemming, L. (2017). Self-compassion as self-care: a simple and effective tool for counselor educators and counseling students. Journal of Creativity in Mental Health, 12, 1-13.

Onur, A., Sahina, E., \& Tekkaya, C. (2012). An investigation on value orientations, attitudes and concern towards the environment: the case of Turkish junior school students. Environmental Education Research, 18(2), 271-297.

Shchetinina, A.M. (2000). Diagnosis of social development of a child. Velikiy Novgorod: NOVGU im. Yaroslava Mudrogo.

Vekiri, I., \& Chronaki, A. (2008). Gender issues in technology use: Perceived social support, computer self-efficacy and value beliefs, and computer use beyond school. Computers \& Education, 51(4), 392-1404.

Verplanken, B., \& Holland, R.W. (2002). Motivated decision making: Effects of activation and self-centrality of values on choices and behavior. Journal of Personality and Social Psychology, 82(3), 434-447. 